2. Furthermore, to study the confound factors which increase the sensitivity of this test in order to reduce unnecessary culture of urinanalysis.

A total of eighty patients admitted to Pediatrics wards at AlAdan Hospital over a period of four months were found to have a positive nitrite test in routine urinanalysis. only $38(47 \%)$ were significantly positive (pure bacterial growth of $>=100000 \mathrm{CFU} / \mathrm{ml}$ ) Other studied confound factors such as fever, urinary symptoms, rigors, family history and past history of UTI, history of nocturnal enuresis or constipation were only significant if taken in combination (of those with positive urine cultures $97 \%$ had more than one factor). Urinary leukocyte count was significantly elevated ( $>10 /$ hpf) in $90 \%$ of the patients with a positive urine cultures. Our data indicates that ordering urine culture in patients with a positive nitrite in urinanalysis should be restricted only to those who also have elevated urinary leukocyte esterase and those who have one or more risk factor for UTI.

\section{DISTRIBUTION OF INTESTINAL PARASYTES IN A PEDIATRICS CLINIC IN 3 YEARS' PERIOD}

doi:10.1136/archdischild-2012-302724.0836

${ }^{1} \mathrm{M}$ Ugras, ${ }^{20}$ Miman, ${ }^{3} \mathrm{~F}$ Alpay. ${ }^{1}$ Pediatrics, Yeditepe Univ Medical Faculty, Istanbul; ${ }^{2}$ Microbiology, ${ }^{3}$ Pediatrics, Kocatepe University Medical Faculty, Afyonkarahisar, Turkey

Aim Intestinal parasitic infections are frequently seen in developing countries. Clinical findings such as abdominal pain, anal itching, salivation during sleep, and nazal itching are related with the prevalence of parasitic infection. The reported ranges from different cities in Turkey vary between $4.4 \%$ and $44.6 \%$. We aimed to look for the prevalence of intestinal parasytes in children who were brought to our clinic in a 3-years' period.

Material and method The laboratory and clinical data of the children who were admitted to the Department of Pediatrics between January 2010 and December 2012 were retrospectively evaluated. Age, major complaint of the children were noted.

Results A total of 1790 stool samples were studied and 116 samples $(6.48 \%)$ had intestinal parasites. There were 1712 children aged between 5 month and 17 years. The complaints were abdominal pain, failure to thrive and anal itching. Intestinal parasites were Giardia intestinalis 33 (1.84\%), Blastocystis hominis 52 (2.9\%), Enterobius vermicularis 13 (0.72\%), Entamoeba coli 17 (0.95\%), Maya was found in 95 stool samples (5.3\%). Multiple parasites were seen in 1 (0.05\%) sample (Blastocystis hominis, Entamoeba coli).

Conclusion Intestinal parsites are frequently seen in developing countries. The mean vales are reported as $12 \%, 10 \%$ and $19 \%$ from different cities in Turkey, ranging between $4.4 \%$ and $44.6 \%$. We found $6.5 \%$ intestinal parasites among children. The most common parasite was $B$. hominis and $G$. intestinalis. The prevalence and type of the parasites are similar when compared with the studies made in our country.

\section{VIEWS OF HEALTH CARE STAFF ABOUT PANDEMIC INFLUENZA VACCINE PRIOR TO PANDEMICS}

doi:10.1136/archdischild-2012-302724.0837

${ }^{1} \mathrm{~A}$ Ozkaya-Parlakay, ${ }^{1} \mathrm{AH}$ Abdulmumin, ${ }^{1} \mathrm{~A}$ Kara, ${ }^{1} \mathrm{AB}$ Cengiz, ${ }^{2} \mathrm{E}$ Ince, ${ }^{2} \mathrm{E}$ Ciftci, ${ }^{3} \mathrm{H}$ Tezer, ${ }^{2} \mathrm{H}$ Ozdemir, ${ }^{3} \mathrm{E}$ Celiker. ${ }^{1}$ Pediatric Infectious Diseases, Hacetepe University, Faculty of Medicine; ${ }^{2}$ Pediatric Infectious Diseases, Ankara University; ${ }^{3}$ Pediatric Infectious Diseases, Ankara Hematology and Oncology Training Hospital, Ankara, Turkey

Pandemic influenza vaccine, is important in many aspects for health care staff. Our questionnaire was conducted in 3 different centers to evaluate view of health care staff for pandemic influenza vaccine prior to epidemics. Our questionnaire was conducted with health care workers having possibility of direct exposure to patients including 182 doctors, 158 nurses and 54 management staff with a total of
394 people. The center of the staff, position, period of Office, whether she/he had seasonal influenza vaccine, whether he/she plans to get pandemic influenza vaccine and reason for this was questioned and noted. Of the 394 healtcare staff included in our study 221 were working in Hacettepe University Hospital,102 were in Ankara University Hospital, 71 were in Ankara Hematology and Oncology Hospital. Of the people enrolled in our study 259 were planning to get pandemic influenza vaccine while 135 were not. Of the staff planning to get pandemic influenza vaccine $77.6 \%$ were planning because they thought they were in risc group, $22.4 \%$ were planning because there was pandemics. Of the staff not planning to get pandemic influenza vaccine $23 \%$ were not taking vaccine because they did not want be guinae pig, 30.9\% were concerned with side affects of vaccine, $46 \%$ had concerns as vaccine was too new. In case of group vaccination declaration of the specialists and determination of health care authorities is very important, so declarations about vaccine should adhere to this aspect. Other wise even in risc groups acceptance of the vaccine would be in lower rates.

\section{ABDOMINAL TUBERCULOSIS DUE TO MYCOBACTERIUM BOVIS}

doi:10.1136/archdischild-2012-302724.0838

G Lozano Sánchez, E Núñez Cuadros, D Moreno Pérez, VM Candón Moreno, B Carazo Gallego, A Urda Cardona. Paediatric, Hospital Carlos Hava, Málaga, Spain

Background Currently, disease related to Mycobacterium bovis $(\mathrm{MB}$ ) is rare in Spain (less than 1\% of tuberculosis cases). However it's more frequent in undeveloped countries where the way to become infected is usually by raw milk consumption.

Methods We describe the epidemiologic, clinical and therapeutic characteristics of 5 patients with abdominal tuberculosis due to $\mathrm{MB}$.

Results Five children ( 3 male) emanating from Morocco with ages between 2 and 6 years, all of them had fever, abdominal pain and constitutional syndrome. Three of them used to drink raw goat's or cow's milk. In 2 cases tuberculin skin test was over than $12 \mathrm{~mm}$. There was open surgery (3) or laparoscopy (2) for realization of lymph node biopsy or drainage of abscess. The diagnosis was through culture and PCR in drained material and/or lymph node. Associated complications were: intraabdominal bacterial infection (4), esteroraceus fistula (2), intestinal occlusion (3). All patients were immunocompetent, except one case with histocompatibility complex class II molecules deficiency. Empirical treatment consisted in isoniazid and rifampicin for 6 months, amikacin (3) or ethambutol (2) during 1 or 2 months respectively. One patient developed a Dress syndrome related to rifampicin. All patients received antituberculous drugs during 6 months, except two patients who needed extended therapy.

Conclusion Treatment for abdominal tuberculosis must be medical, and surgery should be used only in serious complications or biopsy. Although the clinical presentation is often very similar from the produced by $M$. tuberculosis, empirical therapy avoiding pyrazinamide should be started if $M B$ is suspected.

\section{VIEW OF PREGNANT WOMEN ABOUT PANDEMIC INFLUENZA VACCINE PRIOR TO PANDEMIA}

doi:10.1136/archdischild-2012-302724.0839

'A Ozkaya-Parlakay, ${ }^{20}$ Kara, ${ }^{1} \mathrm{~A}$ Kara, ${ }^{20}$ Ozyuncu. 'Pediatric Infectious Diseases, 2Ubstetrics and Gynecoligy, Hacetepe University, Faculty of Medicine, Ankara, Turkey

Afterwards pregnant women were detected to have serious complications with pandemic A (H1N1) virus in 2009, priority of their vaccination was accepted. Thus our study was planned to estimate the view of pregnant women about pandemic influenza vaccine to prevent disease admitting to a reference hospital having patients all 
over the country, Hacettepe University Medical Faculty Obstetrics and Gynecology Department, Obstetrics Unit, in October 2009, just before pandemic influenza vaccine is implemented clinically to evaluate the perception of vaccine. Our study was conducted as applying questionaire to 86 pregnant women admitting to Hacettepe University Medical Faculty Obstetrics and Gynecology Department, Obstetrics Unit. Age, trimestr and gravida of pregnancy, seasonal influenza vaccine status, whether she finds pandemic influenza vaccine necessary was noted after questionnaire. Pregnant women contributing to our questionaire were between 18-39 years of age, 13 of them were in first trimestr, 31 were in second trimestr, 42 were in third trimestr. Out of 86 pregnant women 66 expresses that they did not find it necessary to have pandemic influenza vaccine. In our study it was found that pregnant women find pandemic influenza vaccine necessary with a percentage of $23.3 \%$ and although the study population is small, finding it necessary was not effected by mother's age, trimestr of pregnancy, gravida of pregnancy. The only factor demonstrated to effect the acceptance of pandemic influenza vaccine was having seasonal influenza vaccine. It is very important to inform this group having the major risk, to remind and advise the vaccine in routine controls.

\section{NEUROBRUCELLOSIS IN CHILDHOOD: FOUR NEW CASES AND A REVIEW OF THE LITERATURE}

doi:10.1136/archdischild-2012-302724.0840

HA Ayyash. Pediatrics Department, Islamic Hospital, Amman, Jordan

Background and Aims Neurobrucellosis accounts for $<1 \%$ of cases of brucellosis in children and has a broad range of clinical manifestations. In this report we describe our experience regarding the epidemiological, clinical, laboratory and therapeutic findings in four children with neurobrucellosis.

Methods During the past nine years we treated four children with neurobrucellosis in the pediatric department, of Islamic Hospital Amman, Jordan.

The diagnosis was based on epidemiological evidence of brucellosis, as well as Serum \& cerebrospinal fluid(CSF) serology, quantitative changes in C.S.F and favorable response to treatment. Therapy consisted of combinations of two or three of the following drugs for three months: rifampin, gentamycin, streptomycin and trimethoprim-sulfamethoxazole.

Results The main presenting clinical features included fever, neck stiffness. Neurologic signs appeared during the active phase in two patients and later in ther two patients. The interval from onset of symptoms to diagnosis was from 3 days to 5 months. The mean age of children was 7.2 years, and the male: female ratio $3: 1$.

Brucella anti bodies were detected in all sera with levels $\geq 320$ in two cases and $\geq 1280$ in the other two cases.

Cultures in the blood or C.S.F for brucella were unrevealing in all patients.

Cerebrospinal fluid showed: lymphocytosis (500-2160) $\mu \mathrm{l}$ in all cases, elevated proteins in three cases, decreased glucose in two and a Brucella microaggglutination test titre of of $\geq 1: 80$ in 2 cases.

Treatment was successful in all patients after 12 months.

Conclusion We suggest that neurobrucellosis should be considered when neurological manifestations ensues with unknown etiology in endemic areas.

\section{SALMONELLA TYPHI SEPTICEMIA WITH ACUTE RENAL FAILURE IN A 11 YEAR OLD BOY SECONDARY TO PROLIFERATIVE GLOMERONEPHRITIS: A CASE REPORT}

doi:10.1136/archdischild-2012-302724.0841

GS Dhooria, HS Bains, D Bhat. Pediatrics, Dayanand Medical College \& Hospital, Ludhiana, India
An 11 year-old boy was admitted to our hospital because of high fever, gross hematuria and pain in abdomen. He also had hypertension, nephrotic range proteinuria with renal failure, for which hemodialysis was required. Salmonella Typhi was isolated from blood culture and was diagnosed to have typhoid fever. In view of low C3 levels, renal biopsy was done, showed evidence of proliferative glomerulonephritis. On discharge, he had mildly deranged renal function with persistence of gross hematuria and proteinuria which gradually resolved over a period of one year. Renal involvement with enteric fever is noticed only in $2-3 \%$ cases. The common complications of typhoid related to the urinary tract include cystitis, pyelitis, pyelonephritis, and mild proteinuria. Few cases have been reported of acute nephritic syndrome in typhoid fever requiring renal replacement therapy. Here, we report a case of Salmonella typhi septicemia associated with acute renal failure secondary to proliferative glomeronephritis.requiring renal replacement therapy.

\section{SLAPPED-CHEEK INFECTIONS IN CHILDREN WITH SEVERE COMPLICATIONS IN PRESCHOOL CHILDREN}

doi:10.1136/archdischild-2012-302724.0842

${ }^{1}$ A Bajraktarevic, 'E Selimic, 'M Miokovic, 'S Trhulj Putica, 'L Kumasin, 'A Skopljak, ${ }^{1} \mathrm{~N}$ Dizdarevic Kreso, ${ }^{2} \mathrm{~B}$ Djukic, ${ }^{3} \mathrm{~A}$ Selimovic, ${ }^{3} \mathrm{E}$ Mujicic Selimovic, ${ }^{4} \mathrm{~A}$ Drnda, ${ }^{5} \mathrm{Z}$ Jatic, ${ }^{6}$ Suljevic, ${ }^{7 J}$ Gutic, ${ }^{7} \mathrm{M}$ Ridzal. 'Pediatrics Department, Public Health Institution of Sarajevo Canton; ${ }^{2}$ Pediatrics Department, First Medical Aid; ${ }^{3}$ Pulmonology Department, Pediatrics Clinic; ' ${ }^{4}$ ediatrics Departrment, Infectious Clinic Sarajevo; ${ }^{5}$ Department for Family Medicine, Medical Faculty Sarajevo; ${ }^{6}$ Laboratory Biochemistry Department, Clinical Medical Center; ${ }^{\top}$ Perinatology Department, General Hospital Sarajevo, Sarajevo, Bosnia-Herzegovina

Background The most common illness caused by parvovirus B19 infection is 'fifth disease', a mild rash illness that occurs most often in children. The infection often results in no obvious illness. It commonly infects children and typically causes a mild rash that may resemble a "slapped-cheek". Other symptoms that can occur include joint pain (arthralgia), fever and general flu-like symptoms.

Methods Children who are at risk of severe parvovirus complications might benefit from blood tests that can help determine if they're immune to parvovirus or if they've recently become infected. Most cases of slapped cheek syndrome diagnosed by making a visual examination of the distinctive rash. No further testing was usually required in children during three years period 20092011 in capital town of Bosnia, Sarajevo.

Results Parvovirus infection in children with anemia may stop the production of red blood cells and cause an anemia crisis. Children with severe anemia may need to be hospitalized and receive blood transfusions. Percentage of hospitalized of parvivirus infections in children with anaemia is $19 \%$ in Bosnian preschool children, what is only less $1 \%$ of complications in this disease.

Conclusions Fifth disease can cause fetal anemia, which if undetected can have severe consequences. Several days after the appearance of early symptoms, a distinctive bright red facial rash may appear usually on both cheeks. In most children, parvovirus infection is mild and requires little treatment. Slapped cheek is actually the Parvovirus B19 that only affects humans, especially younger children.

\section{REFRACTORY CASE OF KAWASAKI DISEASE WITH HEMOLYTIC ANEMIA: CORRELATION WITH MYCOPLASMATIC INFECTION}

doi:10.1136/archdischild-2012-302724.0843

M Kousouri, E Roumbani, C Koutsaftiki, A Balaska, K Papantzimas, N Myriokefalitakis. 1st Pediatric Department, Penteli's Children's Hospital, Athens, Greece

Introduction Kawasaki Disease $(\mathrm{KD})$ is a systematic disease, usually affecting children 6months-5 years old. Optimal therapy is 